\title{
Investigating age-induced differentially expressed genes and potential molecular mechanisms in osteosarcoma based on integrated bioinformatics analysis
}

\author{
JIAN-SHENG WANG ${ }^{1}$, MING-YUE DUAN ${ }^{2}$, YONG-SHENG ZHONG ${ }^{3}$, XUE-DONG $\mathrm{LI}^{4}$, \\ SHI-XIN DU ${ }^{4}$, PENG XIE ${ }^{4}$, GUI-ZHOU ZHENG ${ }^{4}$ and JING-MING HAN ${ }^{1}$ \\ ${ }^{1}$ Department of Orthopedics Ward II, Shenzhen Children's Hospital, Shenzhen, Guangdong 518038; \\ ${ }^{2}$ Shanxi Institute of Pediatric Diseases, Xi'an Children's Hospital, Xi'an, Shanxi 710043; ${ }^{3}$ Department of Neurosurgery, \\ The First Affiliated Hospital of Shantou University Medical College, Shantou, Guangdong 515041; \\ ${ }^{4}$ Department of Orthopedics, The Third Affiliated Hospital of Shenzhen University \\ Health Science Center, Shenzhen, Guangdong 518000, P.R. China
}

Received May 25, 2018; Accepted January 22, 2019

DOI: $10.3892 / \mathrm{mmr} .2019 .9912$

\begin{abstract}
Osteosarcoma (OS) is the most common primary bone malignancy. It predominantly occurs in adolescents, but can develop at any age. The age at diagnosis is a prognostic factor of OS, but the molecular basis of this remains unknown. The current study aimed to identify age-induced differentially expressed genes (DEGs) and potential molecular mechanisms that contribute to the different outcomes of patients with OS. Microarray data (GSE39058 and GSE39040) obtained from the Gene Expression Omnibus database and used to analyze age-induced DEGs to reveal molecular mechanism of OS among different age groups ( $<20$ and $>20$ years old). Differentially expressed mRNAs (DEMs) were divided into up and downregulated DEMs (according to the expression fold change), then Gene Ontology function enrichment and Kyoto Encyclopedia of Genes and Genomes pathway analysis were performed. Furthermore, the interactions among proteins encoded by DEMs were integrated with prediction for microRNA-mRNA interactions to construct a regulatory network. The key subnetwork was extracted and Kaplan-Meier survival analysis for a key microRNA was performed. DEMs within the subnetwork were predominantly involved in
\end{abstract}

Correspondence to: Dr Jing-Ming Han, Department of Orthopedics Ward II, Shenzhen Children's Hospital, 7019 Yitian Road, Futian, Shenzhen, Guangdong 518038, P.R. China

E-mail: 279984031@qq.com

Abbreviations: DEGs, differentially expressed genes; DEMs, differentially expressed mRNAs; DEMis, differentially expressed microRNAs; GO, Gene Ontology; BP, biological process; CC, cellular component; MF, molecular functions

Key words: osteosarcoma, age, Gene Expression Omnibus data, integrated bioinformatics, regulatory network 'ubiquitin protein ligase binding', 'response to growth factor', 'regulation of type I interferon production', 'response to decreased oxygen levels', 'voltage-gated potassium channel complex', 'synapse part', 'regulation of stem cell proliferation'. In summary, integrated bioinformatics was applied to analyze the potential molecular mechanisms leading to different outcomes of patients with OS among different age groups. The hub genes within the key subnetwork may have crucial roles in the different outcomes associated with age and require further analysis.

\section{Introduction}

Osteosarcoma (OS), the most common primary malignancy of bone, can occur at any age, but predominantly develops in adolescents, with $\sim 400$ newly diagnosed cases each year in America (1). OS accounts for $\sim 60 \%$ of all bone malignancies diagnosed in patients $<20$ years old $(2,3)$. Several studies reported that disease stage, gender, age, pathology subtypes and primary tumor site of OS result in differences in survival rate, with younger patients exhibiting significant better prognosis and higher survival rate than the elderly $(2,4)$. With improved chemotherapy outcomes in the 1980s, the 5-year event-free survival was significantly increased, whereas it has remained stable without any significant improvement in the past 20 years (5-7). Uncovering the tumorigenesis and progression mechanisms is crucial for developing effective molecular targeted therapy. In the past decades, efforts have been made to clarify the molecular basis of OS. Substantial progress has been made using gene expression profiles of OS to reveal the molecular pathogenesis of OS. Age is a prognostic factor of OS insofar as we know; however, few studies have investigated the underlying molecular mechanisms, which may contribute to various outcomes.

Original microarray datasets of 36 OS samples from patients aged $<20$ and 6 matched samples aged $>20$ (GSE39058) (8), and 58 OS samples from patients aged $<20$ and 7 matched samples from patients aged $>20$ from human 
non-coding RNA profiling data(GSE39040) (8), were obtained from the human expression database Gene Expression Omnibus (GEO; https://www.ncbi.nlm.nih.gov/geo/). Differentially expressed genes (DEGs) between OS cases aged $<20$ and matched cases aged $>20$ were screened using $\mathrm{R}$ software limma package (9). Gene function analysis, including Gene Ontology (GO) and KOBAS-Kyoto Encyclopedia of Genes and Genomes (KEGG) pathways, were performed using the differentially expressed mRNAs on Metascape (http://metascape.org/gp/). A regulatory network was constructed by combining the protein-protein interaction (PPI) network with microRNA (miRNA)-mRNA interactions to reveal the potential molecular differences between the different age groups. Kaplan-Meier survival analysis was also performed to clarify our result. In conclusion, the regulatory networks were constructed and the potential important key signaling pathways were analyzed by applying bioinformatics tools to investigate different molecular mechanism of OS among different age groups.

\section{Materials and methods}

Microarray data. Microarray data was retrieved from the GSE39058 and GSE39040 GEO (https://www.ncbi.nlm.nih. gov/geo). The mRNA expression of $36 \mathrm{OS}$ samples from patients aged $<20$ and 6 matched samples from patients aged $>20$ obtained from GSE39058, based on the GPL14951 Illumina HumanHT-12 WG-DASL V4.0 R2 expression BeadChip (Illumina, Inc., San Diego, CA, USA). Similarly, a total of 58 OS samples from patients aged $<20$ and 7 matched samples from patients aged $>20$ were available in GSE39040, based on the GPL15762 Illumina Human v2 MicroRNA Expression BeadChip.

Data processing. Following background correction and quartile data normalization of the original microarray data by using the robust multi-array average algorithm, probes without a corresponding gene symbol were filtered and the average value of gene symbols with multiple probes was calculated. The selected data from GSE39058 and GSE39040 were compared using R software limma package (http://www.bioconductor. org/packages/release/bioc/html/limma.html) to determine the differentially expressed mRNAs and miRNAs (10). With a threshold of $\mathrm{P}<0.05$ and $\log _{2}$ (fold change) $\mid>1$, volcano plot filtering was performed by using $\mathrm{R}$ software ggplot 2 package v2.2 (11) to identify the DEGs with statistical significance between two groups. Hierarchical clustering and combined analyses were performed for the DEGs. The differentially expressed mRNAs and miRNAs obtained were described as age-induced osteosarcoma DEMs and DEMis.

GO and KEGG pathway enrichment analysis of DEMs. DEMs were converted to their corresponding Homo sapiens Entrez gene IDs using the latest database (ncbi.nlm.nih.gov/gene; updated on 2018-01-01); pathway and process enrichment analysis of DEMs was performed using Metascape (metascape. org). All genes in the genome were used as the enrichment background. Terms with $\mathrm{P}<0.05$ were statistically significant. Those with a minimum count of 3 and enrichment factor $>1.5$ (enrichment factor is the ratio between observed count and the count expected by chance) were collected, and grouped into clusters based on their membership similarities. More specifically, P-values were calculated based on accumulative hypergeometric distribution, q-values were calculated using the Benjamini-Hochberg procedure to account for multiple testing. $\kappa$ scores were used as the similarity metric when performing hierachical clustering on the enriched terms and then sub-trees with similarity $>0.3$ were considered a cluster. The most statistically significant term within a cluster was selected to represent the cluster.

Regulatory network construction and analysis. The Search Tool for the Retrieval of Interacting Genes/Proteins (STRING) database (string-db.org/) was used to explore the interactions among proteins encoded by the DEMs, including known proteins interactions and predicted interactions. The results were downloaded from the STRING database as a TSV file, the PPI pairs with selected larger scores were imported to the Cytoscape tool v3.4.0 (cytoscape.org/) to establish PPI network. The regulatory relationship between genes were analyzed through topological property of computing network including the degree distribution of network by using the CentiScaPe2.2 app within Cytoscape (12). The interaction between DEMs and DEMis was predicted using miRwalk v3.0 (mirwalk.umm.uni-heidelberg.de/) with a threshold of energy $<-20$, and only the interactions of miRNA and mRNA with opposite expression were included. Furthermore, the regulatory network was constructed by combining the PPI network with the predicted interactions between DEMs and DEMis. The subnetwork was extracted from the whole PPI network using the MCODE app v1.5.1 (13).

Kaplan-Meier survival analysis. In the present study, the prognostic value of the genes was confirmed by Kaplan-Meier survival analysis based on the clinical information from the GSE39040 dataset using SPSS 16.0 (SPSS, Inc., Chicago, IL, USA). For statistical analysis, the patients were divided into halves based on gene expression values. Specifically, patients with the expression values greater than the median value were classified as the high expression group and others classified as the low expression group. Log rank test (Mantel-Cox test) was used to evaluate the prognostic value of the genes.

\section{Results}

Data processing and DEG screening. DEMs of GSE39058 were obtained using $\mathrm{R}$ software limma package with $\mid \log _{2}$ (fold change) $\mid>1$ and $\mathrm{P}<0.05$ as the cut-off point. In OS samples from patients aged $<20$, there were 1,476 up-regulated DEMs, corresponding to 1,525 probes and 637 downregulated DEMs detected by 650 probes in OS samples from patients aged $<20$ compared with OS samples from patients aged $>20$. The top 10 most significantly upregulated genes were SNORA17, SNORD3C, CRY1, TSEN34, NDUFA12, CNTFR, CPE, PTGS2, NKD2 and EGFL6. The top 10 most significantly downregulated genes were PPP2R2B, DSCR8, ALAS2, GPR114, NPLOC4, PDE7B, NCOA2, MRFAP1L1, LIG3 and RUNX1. Similarly, there were 15 DEMis in GSE39040, including 11 downregulated and 4 upregulated DEMis OS samples from patients aged $<20$ compared with OS samples 

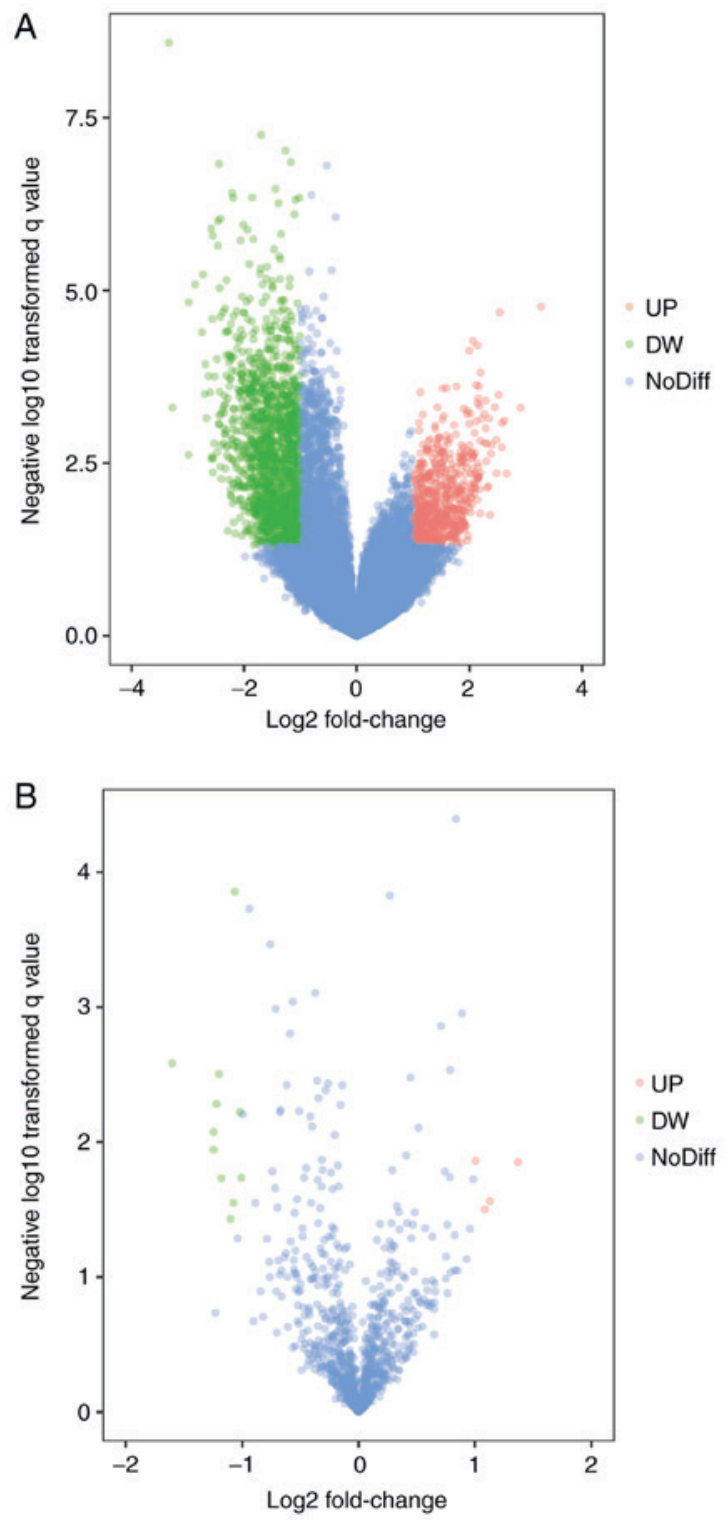

Figure 1. Differentially expressed genes between two sets of samples. (A) GSE39058 data,(B) GSE39040 data. The red points represent upregulated genes screened on the basis of |fold changel $<2.0$ and $\mathrm{P}<0.05$. The green points represent downregulated genes screened on the basis of Ifold changel $<2.0$ and $\mathrm{P}<0.05$. The blue points represent genes with no significant difference. UP, upregulated; DW, downregulated; NoDiff, no significant difference.

from patients aged $>20$. The DEMis upregulated in OS samples from patients aged $<20$ were hsa-miR-1248, hsa-miR-497, hsa-miR-1201 and hsa-miR-224. The downregulated DEMis were hsa-miR-122, hsa-miR-203, hsa-miR-205, hsa-miR-194, hsa-miR-200c, hsa-miR-183, hsa-miR-142-5p, hsa-miR-182, hsa-miR-549, hsa-miR-202* and hsa-miR-515-5p. The volcano plot of each microarray is presented in Fig. 1 to visualize DEGs. Fig. 2 presented the cluster heatmaps of DEMs and DEMis.

DEMs enrichment analysis. To determine functional enrichment, Metascape was used for GO and KEGG enrichment analysis of DEMs. GO functional enrichments and KEGG pathways of which $\mathrm{P}<0.05$ were selected as significant results (Table I). GO analysis of DEGs includes three functional groups, namely molecular function, biological processes and cellular component. The significant GO enrichment analysis
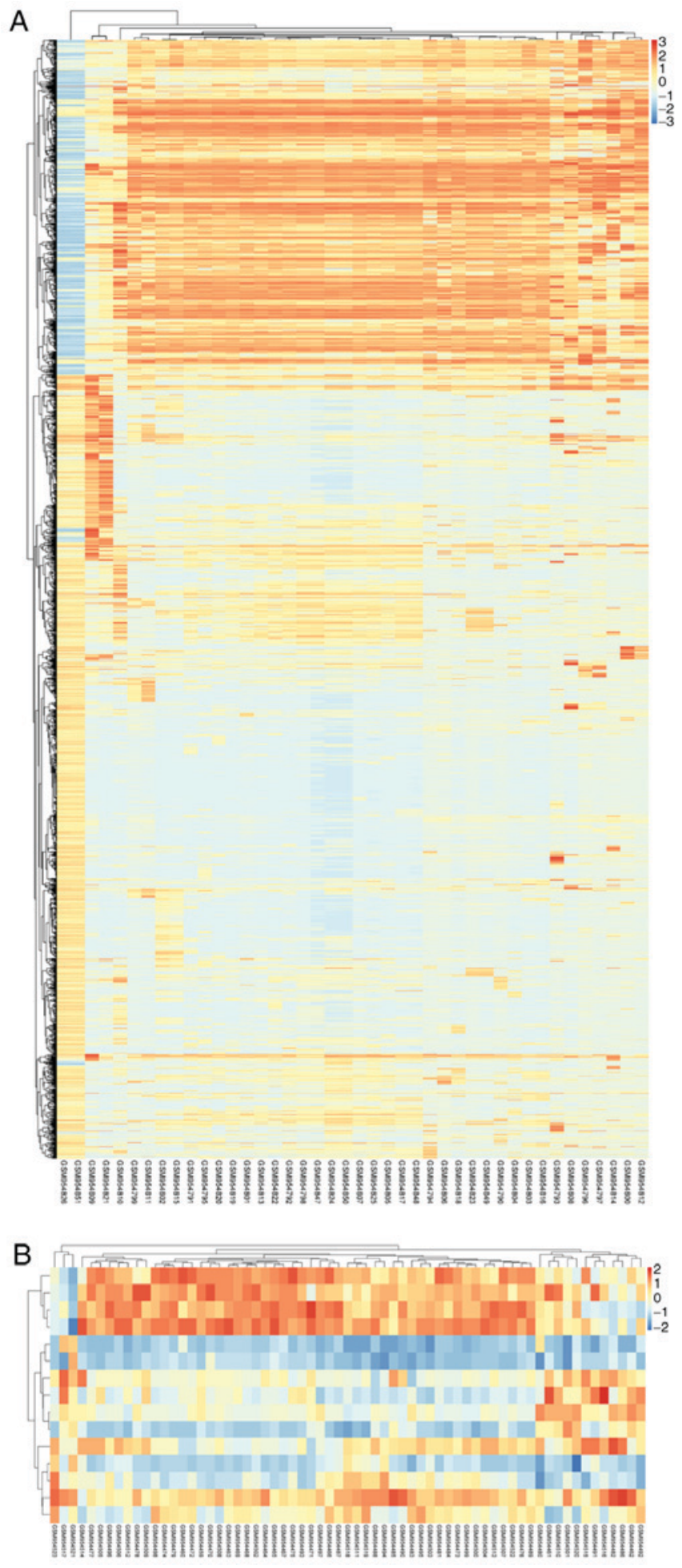

Figure 2. Hierarchical clustering heatmap of DEGs screened on the basis of $\log _{2}$ (fold change) $<2.0$ and $\mathrm{P}<0.05$. (A) GSE39058, (B) GSE39040. Orange indicates that the expression of genes is relatively upregulated; blue indicates that the expression of genes is relatively downregulated, and white indicates no significant difference.

of all DEMs is presented in Fig. 3. GO functional enrichments and KEGG pathway analysis of up- and down-regulated DEMs were also performed using Metascape. The significant results $(\mathrm{P}<0.05)$ are presented in Figs. 4 and 5, with details information in Tables II and III. Upregulated DEMs were predominantly involved in the 'cell growth', 'response to 
Table I. GO and KEGG pathway enrichment analysis for the differentially expressed mRNAs.

\begin{tabular}{|c|c|c|c|c|}
\hline Category & Term & Description & Count & $\log \mathrm{P}$ \\
\hline Canonical pathways & M3468 & NABA ECM REGULATORS & 14 & -6.64807 \\
\hline GO BP & GO:0002275 & Myeloid cell activation involved in immune response & 33 & -15.539 \\
\hline GO BP & GO:0006954 & Inflammatory response & 34 & -12.1737 \\
\hline GO BP & GO:0030316 & Osteoclast differentiation & 13 & -11.0649 \\
\hline GO BP & GO:0007159 & Leukocyte cell-cell adhesion & 21 & -10.3439 \\
\hline GO BP & GO:0002253 & Activation of immune response & 28 & -9.66921 \\
\hline GO BP & GO:0050900 & Leukocyte migration & 23 & -9.04039 \\
\hline GO BP & GO:0019221 & Cytokine-mediated signaling pathway & 28 & -7.7241 \\
\hline GO BP & GO:0070372 & Regulation of ERK1 and ERK2 cascade & 16 & -7.41007 \\
\hline GO BP & GO:0006897 & Endocytosis & 26 & -7.22687 \\
\hline GO BP & GO:0031347 & Regulation of defense response & 27 & -7.11793 \\
\hline GO BP & GO:0002250 & Adaptive immune response & 21 & -7.0625 \\
\hline GO BP & GO:0051046 & Regulation of secretion & 25 & -6.61093 \\
\hline $\mathrm{GO} \mathrm{CC}$ & GO:0005773 & Vacuole & 50 & -24.946 \\
\hline $\mathrm{GO} \mathrm{CC}$ & GO:0044440 & Endosomal part & 21 & -7.19392 \\
\hline KEGG pathway & hsa05323 & Rheumatoid arthritis & 19 & -19.1021 \\
\hline KEGG pathway & hsa04142 & Lysosome & 21 & -19.026 \\
\hline KEGG pathway & hsa04380 & Osteoclast differentiation & 18 & -14.6982 \\
\hline KEGG pathway & hsa05152 & Tuberculosis & 18 & -12.2416 \\
\hline KEGG pathway & hsa05150 & Staphylococcus aureus infection & 8 & -6.94145 \\
\hline
\end{tabular}

GO, Gene Ontology; KEGG, Kyoto Encyclopedia of Genes and Genomes; ECM, extracellular matrix; ERK, extracellular signal regulated-kinase; $\mathrm{BP}$, biological process; $\mathrm{CC}$, cellular component.

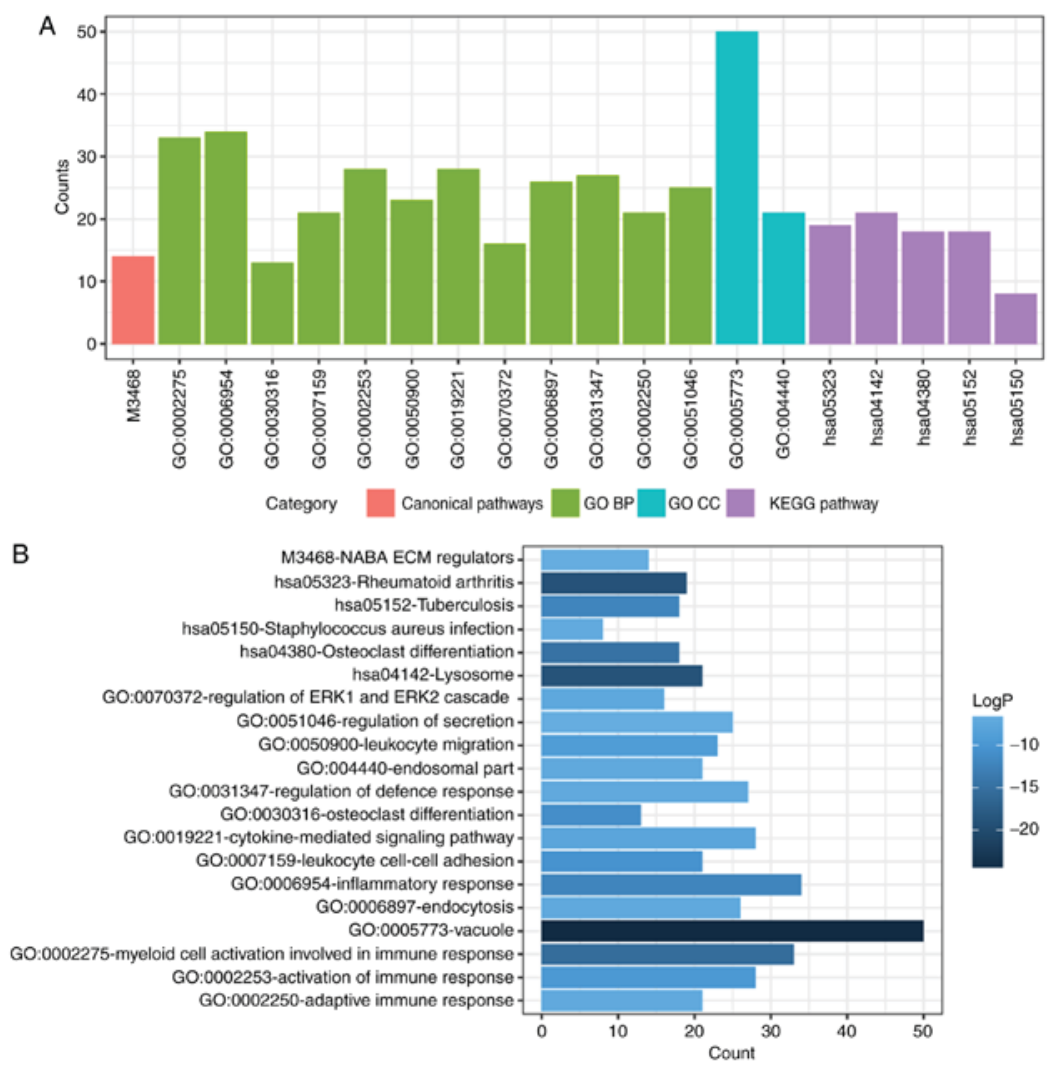

Figure 3. GO and KEGG pathway enrichment analysis of DEMs. (A) GO and KEGG pathway enrichment analysis divided DEMs into four functional groups. (B) Statistical significance of GO and KEGG pathway enrichment of DEMs in different functional groups. DEMs, differentially-expressed mRNAs; GO, Gene Ontology; BP, biological process; CC, cellular component; KEGG, Kyoto Encyclopedia of Genes and Genomes; ECM, extracellular matrix; ERK, extracellular signal regulated-kinase. 


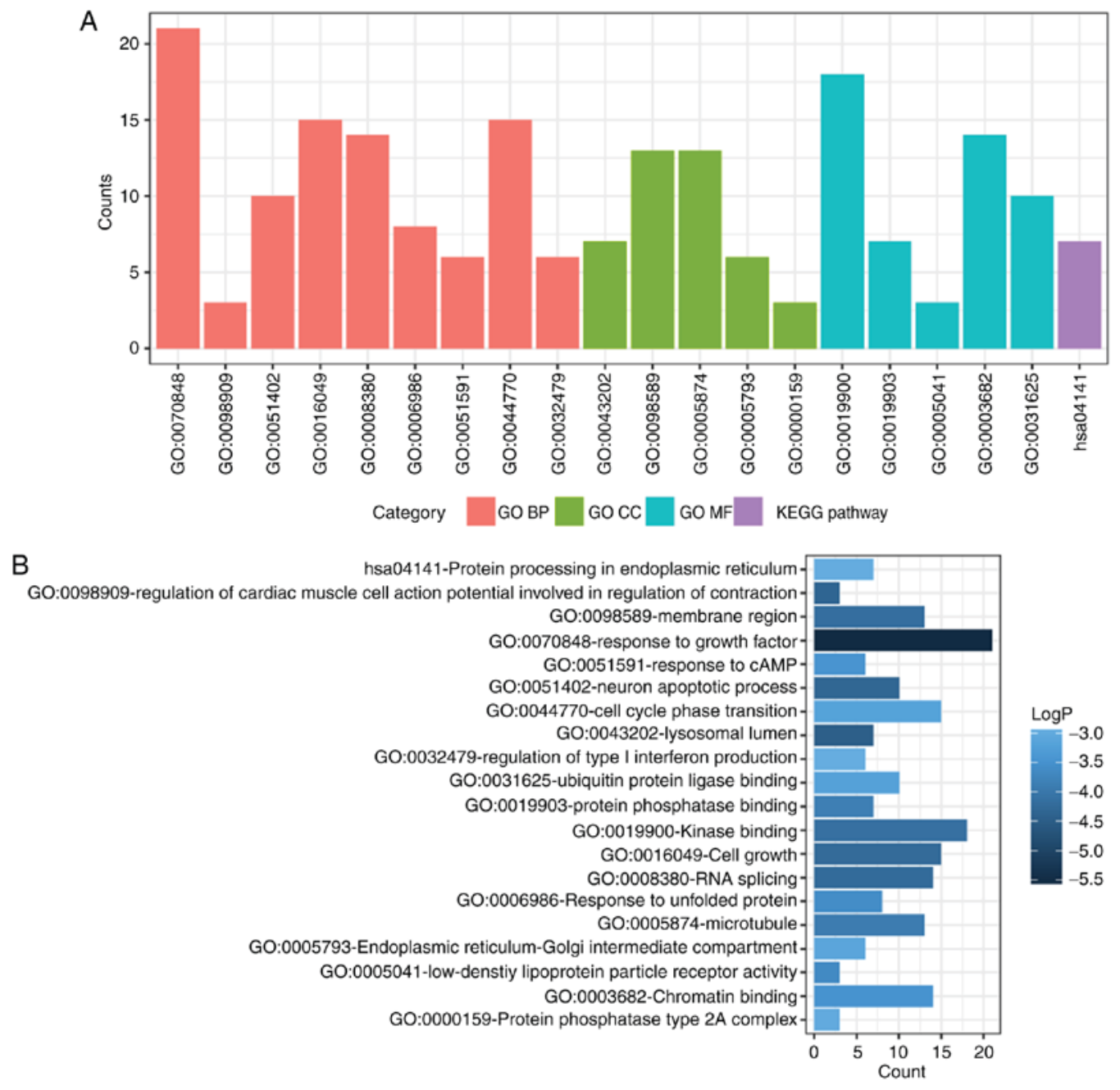

Figure 4. GO and KEGG pathway enrichment analysis of upregulated DEMs. (A) GO and KEGG pathway enrichment analysis divided DEMs into four functional groups. (B) Statistical significance of GO and KEGG pathway enrichment of DEMs in different functional groups. DEMs, differentially-expressed mRNAs; GO, Gene Ontology; BP, biological process; CC, cellular component; MF, molecular function; KEGG, Kyoto Encyclopedia of Genes and Genomes; cAMP, cyclic adenosine monophosphate.

growth factor' and 'neuron apoptotic process' and 'activation of immune response'. The downregulated DEMs were mainly involved in 'cytokine receptor activity', 'phosphoprotein phosphatase activity', 'response to decreased oxygen levels' and 'regulation of stem cell proliferation'.

PPI network. The interactions between proteins encoded by DEMs were downloaded from the STRING database and a PPI network was created using the Cytoscape tool. Additionally, prediction of miRNA-mRNA interactions was integrated into the PPI network. After altering of the color and location of nodes, the PPI network was successfully established (Fig. 6). Furthermore, PPI networks of up- and down-regulated DEMs and their interacting DEMis were also constructed, and the key subnetwork was subsequently extracted using the MCODE app in Cytoscape (Fig. 7). The key significant genes were hsa-miR-497-5p, hsa-miR-203a-5p, hsa-miR-183-5p and hsa-miR-205-5p and the DEMs that they are known to interact with. Of note, up-regulated DEMs within the subnetwork were mainly involved in the ubiquitin-proteasome pathway (UPP), response to growth factors and the regulation of type I interferon production caused by the reduced expression levels of hsa-miR-203, hsa-miR-205 and hsa-miR-183. Furthermore, as a consequence of the overexpression of miR-497, downregulated DEMs within the hub subnetwork were closely associated with response to hypoxia, transport of potassium, activity of synapse, regulation of stem cell proliferation.

Kaplan-Meier survival analysis. The clinical information was obtained from GSE39058 dataset. A total of 65 patients were included in the present study. The log-rank test confirmed that low expression of hsa-miR-203 ( $\mathrm{P}=0.008411$ and $\log 2$ (fold change $=-1.247074$ ) in aged cases was negatively associated with overall survival (Fig. 8A) and positively associated with recurrence rate (Fig. $8 \mathrm{~B}$ ) in patients with OS.

\section{Discussion}

In the current study, microarray data from the GEO database was used to analyze DEGs to reveal differences in molecular mechanism introduced by age. DEMs were divided into two groups by the $\log _{2}$ (fold change), then GO function enrichment and KEGG pathway analysis were performed. Furthermore, an integrated regulatory network was generated by integrating 


\section{A}

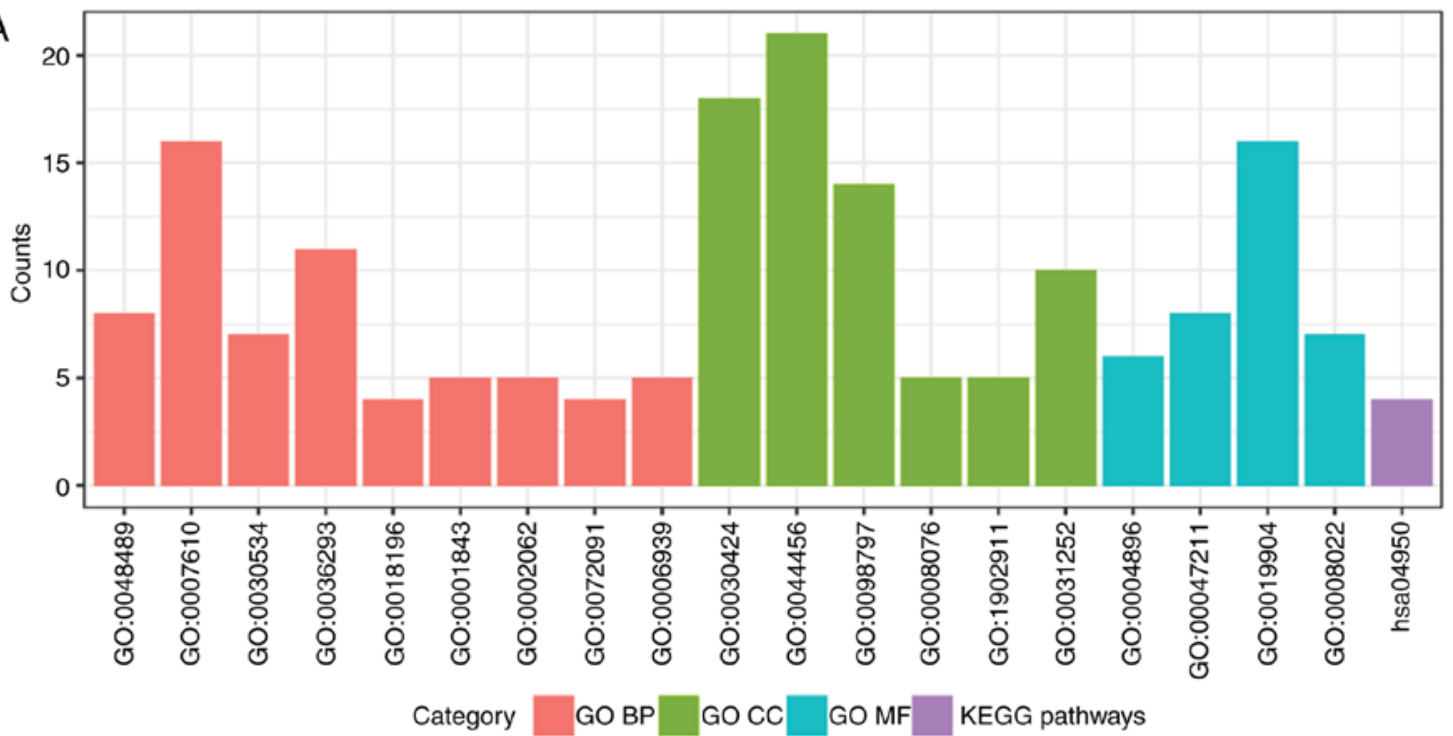

B

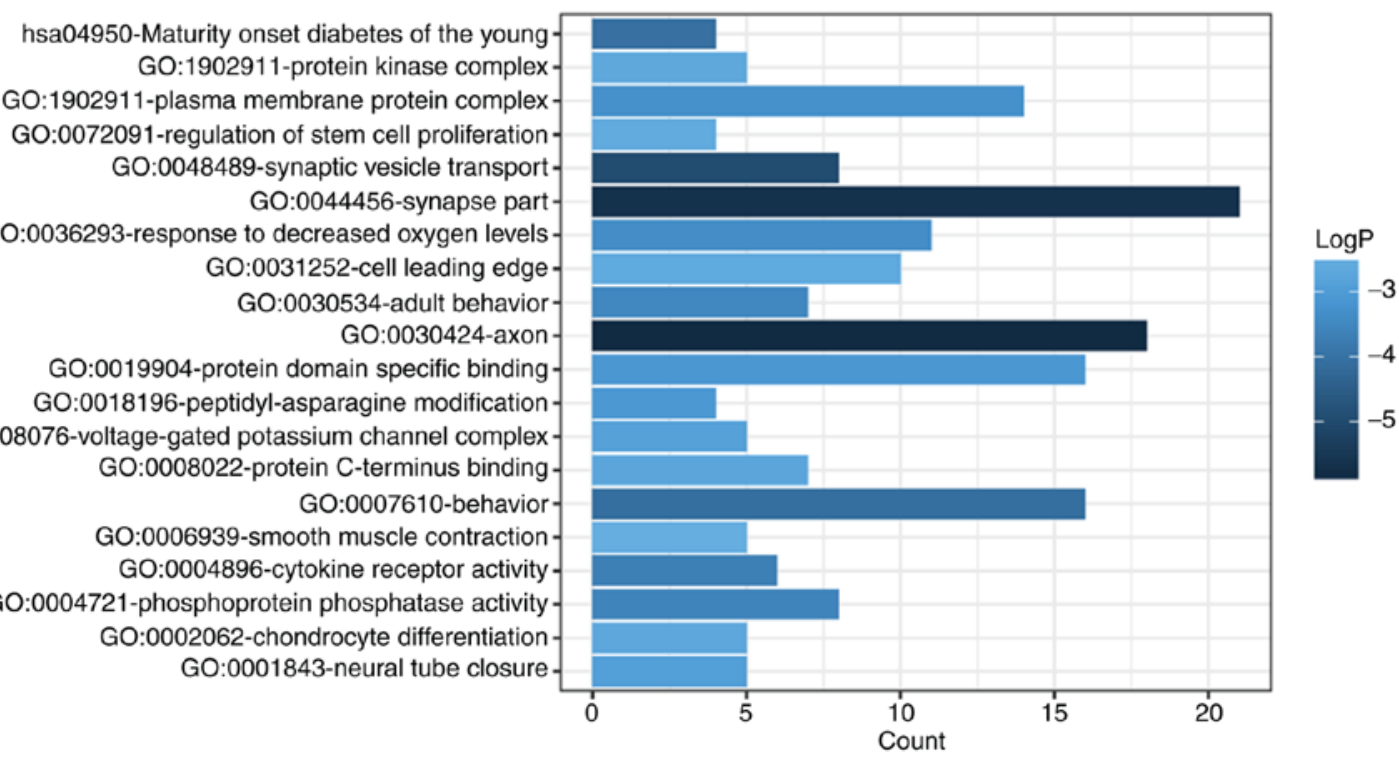

Figure 5. GO and KEGG pathway enrichment analysis of downregulated DEMs. (A) GO and KEGG pathway enrichment analysis divided DEMs into four functional groups. (B) Statistical significance of GO and KEGG pathway enrichment of DEMs in different functional groups. DEMs, differentially-expressed mRNAs; GO, Gene Ontology; BP, biological process; CC, cellular component; MF, molecular function; KEGG, Kyoto Encyclopedia of Genes and Genomes.

the interactions of proteins encoded by DEMs with predictions of miRNA-mRNA interactions. The potential GO functional annotations and the upstream DEMis that may induce the difference in prognosis and survival rate among ages were identified.

From analyzing the key subnetwork combined with the function enrichment analysis, it was apparent that the upregulated DEMs within the key subnetwork were mostly involved in the UPP, response to growth factors and the regulation of type I interferon production. DEMs upregulation may be caused by the reduced expression of hsa-miR-203, hsa-miR-205 and hsa-miR-183. Downregulation of DEMs is potentially caused by upregulated expression of miR-497, and this subnetwork was associated with response to hypoxia, transport of potassium, activity of synapse, regulation of stem cell proliferation.

The UPP is essential for maintaining the homeostasis, as it is responsible for intracellular protein degradation. It has a critical role in many cellular processes, including cell cycle, cell differentiation, apoptosis, anti-apoptosis and tumor development (14-17). The upregulated DEMs enriched in UPP were ubiquitin protein ligase $\mathrm{E} 3$ component $\mathrm{n}$-recognin 2 (UBR2), ubiquitin conjugating enzyme E2 (UBE2) G1,UBE2D3,UBE2Z and Ran GTPase activating protein 1. Of these DEMs, UBE2G1, UBE2D3 and UBE2Z were targets of hsa-miR-203, and hsa-miR-203 was previously demonstrated to be downregulated in OS in patients $<20$ years old in the current study compared with patients $>20$. UBR2, the sole known E3 ubiquitin ligase of the $\mathrm{N}$-end rule pathway, is reported to be upregulated in colon adenocarcinoma and Lewis lung carcinoma (15). UBR2 is also demonstrated to have a pro-apoptotic function by degrading the anti-apoptotic form of tyrosine kinase Lyn, contradicting previous reports of an anti-apoptotic role, indicating that UBR2 might inhibit tumor initiation and progression (14). UBE2D3, a positive prognostic factor in cancer, inhibits cell proliferation and contributes to radiosensitivity, thus improving the survival time (18-20). These findings suggest that UBR2, UBE2G1, 
Table II. GO and KEGG pathway enrichment analysis for the upregulated differentially expressed mRNAs.

\begin{tabular}{|c|c|c|c|c|}
\hline Category & Term & Description & Count & $\log \mathrm{P}$ \\
\hline GO BP & GO:0070848 & Response to growth factor & 21 & -5.5332 \\
\hline GO BP & GO:0098909 & $\begin{array}{l}\text { Regulation of cardiac muscle cell action potential involved } \\
\text { in regulation of contraction }\end{array}$ & 3 & -4.34678 \\
\hline GO BP & GO:0051402 & Neuron apoptotic process & 10 & -4.32756 \\
\hline GO BP & GO:0016049 & Cell growth & 15 & -4.25172 \\
\hline GO BP & GO:0008380 & RNA splicing & 14 & -4.23503 \\
\hline GO BP & GO:0006986 & Response to unfolded protein & 8 & -3.60467 \\
\hline GO BP & GO:0051591 & Response to cAMP & 6 & -3.46953 \\
\hline GO BP & GO:0044770 & Cell cycle phase transition & 15 & -3.20217 \\
\hline GO BP & GO:0032479 & Regulation of type I interferon production & 6 & -2.96638 \\
\hline GO CC & GO:0043202 & Lysosomal lumen & 7 & -4.47707 \\
\hline GO CC & GO:0098589 & Membrane region & 13 & -4.16789 \\
\hline $\mathrm{GO} \mathrm{CC}$ & GO:0005874 & Microtubule & 13 & -3.91148 \\
\hline GO CC & GO:0005793 & Endoplasmic reticulum-Golgi intermediate compartment & 6 & -3.11995 \\
\hline GO CC & GO:0000159 & Protein phosphatase type $2 \mathrm{~A}$ complex & 3 & -3.01052 \\
\hline $\mathrm{GO} \mathrm{MF}$ & GO:0019900 & Kinase binding & 18 & -4.1399 \\
\hline GO MF & GO:0019903 & Protein phosphatase binding & 7 & -3.87788 \\
\hline GO MF & GO:0005041 & Low-density lipoprotein particle receptor activity & 3 & -3.65378 \\
\hline GO MF & GO:0003682 & Chromatin binding & 14 & -3.49506 \\
\hline GO MF & GO:0031625 & Ubiquitin protein ligase binding & 10 & -3.22244 \\
\hline KEGG pathway & hsa04141 & Protein processing in endoplasmic reticulum & 7 & -2.98232 \\
\hline
\end{tabular}

GO, Gene Ontology; KEGG, Kyoto Encyclopedia of Genes and Genomes; cAMP, cyclic adenosine monophosphate; BP, biological process; $\mathrm{CC}$, cellular component; MF, molecular functions.

Table III. GO and KEGG pathway enrichment analysis for the downregulated differentially expressed mRNAs.

\begin{tabular}{|c|c|c|c|c|}
\hline Category & Term & Description & Count & $\log \mathrm{P}$ \\
\hline GO BP & GO:0048489 & Synaptic vesicle transport & 8 & -4.97563 \\
\hline GO BP & GO:0007610 & Behavior & 16 & -4.08991 \\
\hline GO BP & GO:0030534 & Adult behavior & 7 & -3.49616 \\
\hline GO BP & GO:0036293 & Response to decreased oxygen levels & 11 & -3.35132 \\
\hline GO BP & GO:0018196 & Peptidyl-asparagine modification & 4 & -3.07891 \\
\hline GO BP & GO:0001843 & Neural tube closure & 5 & -2.90315 \\
\hline GO BP & GO:0002062 & Chondrocyte differentiation & 5 & -2.67404 \\
\hline GO BP & GO:0072091 & Regulation of stem cell proliferation & 4 & -2.59464 \\
\hline GO BP & GO:0006939 & Smooth muscle contraction & 5 & -2.52575 \\
\hline GO CC & GO:0030424 & Axon & 18 & -5.87109 \\
\hline $\mathrm{GO} \mathrm{CC}$ & GO:0044456 & Synapse part & 21 & -5.66372 \\
\hline $\mathrm{GO} \mathrm{CC}$ & GO:0098797 & Plasma membrane protein complex & 14 & -3.27326 \\
\hline $\mathrm{GO} \mathrm{CC}$ & GO:0008076 & Voltage-gated potassium channel complex & 5 & -2.85904 \\
\hline $\mathrm{GO} \mathrm{CC}$ & GO:1902911 & Protein kinase complex & 5 & -2.65473 \\
\hline $\mathrm{GO} \mathrm{CC}$ & GO:0031252 & Cell leading edge & 10 & -2.62652 \\
\hline GO MF & GO:0004896 & Cytokine receptor activity & 6 & -3.68521 \\
\hline GO MF & GO:0004721 & Phosphoprotein phosphatase activity & 8 & -3.57184 \\
\hline GO MF & GO:0019904 & Protein domain specific binding & 16 & -3.127 \\
\hline GO MF & GO:0008022 & Protein C-terminus binding & 7 & -2.72974 \\
\hline KEGG pathway & hsa04950 & Maturity onset diabetes of the young & 4 & -4.05467 \\
\hline
\end{tabular}

GO, Gene Ontology; KEGG, Kyoto Encyclopedia of Genes and Genomes; BP, biological process; CC, cellular component; MF, molecular functions. 


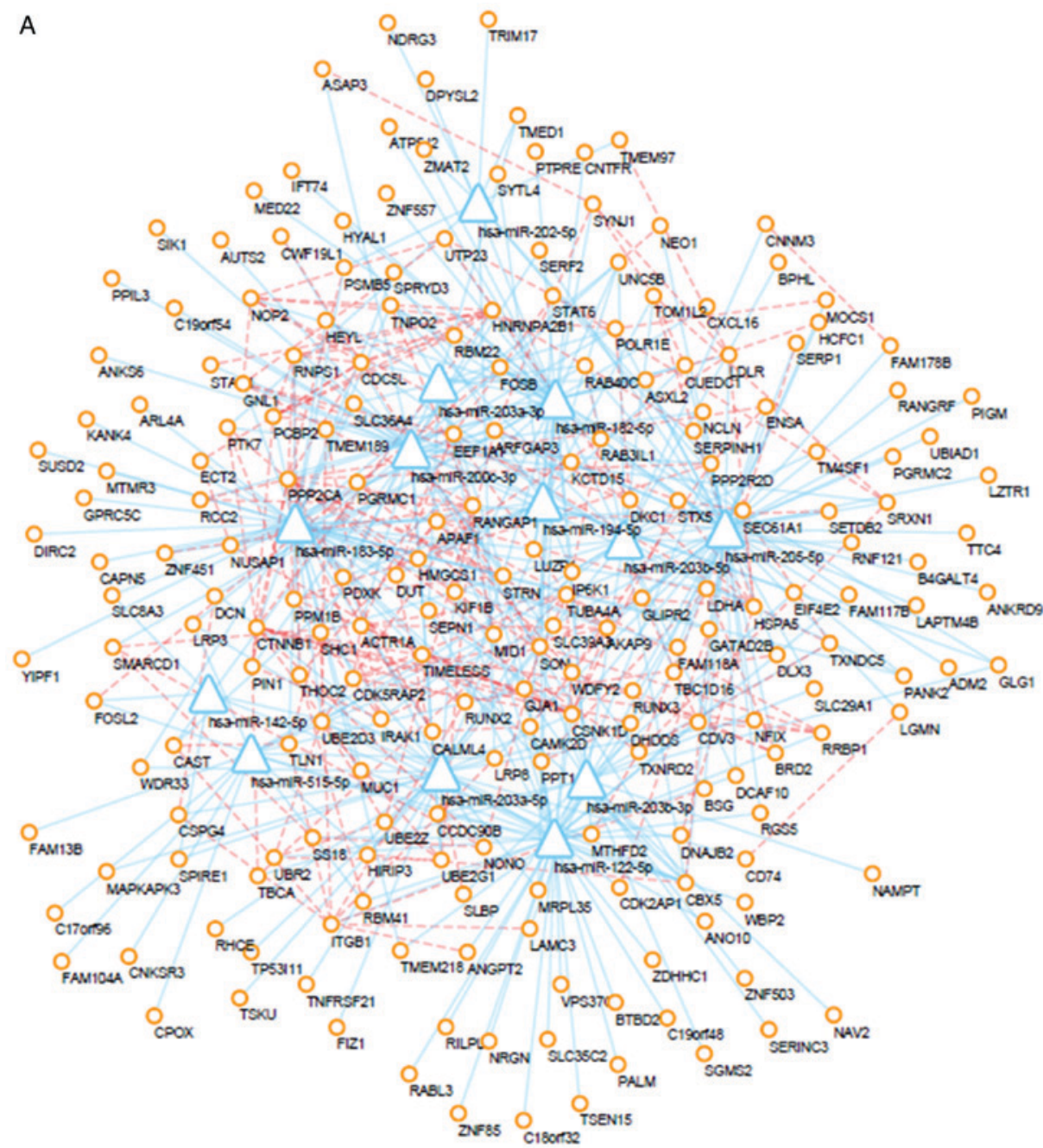

B

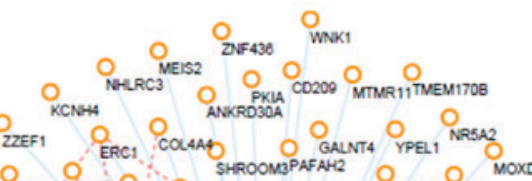

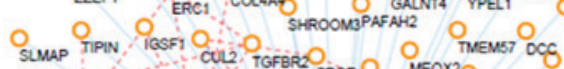

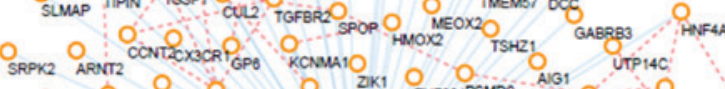

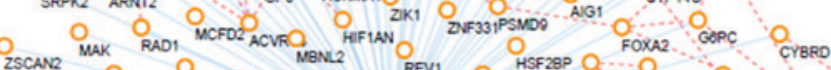

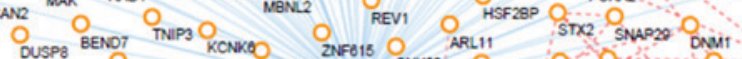

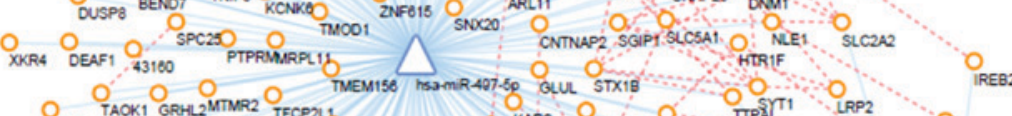

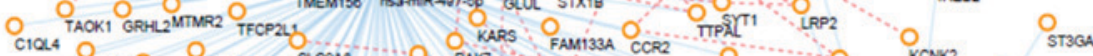

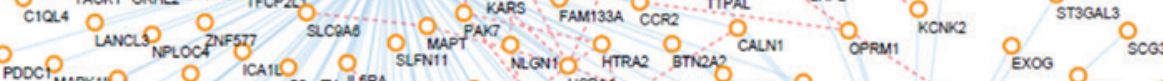

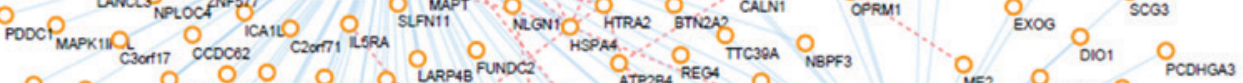

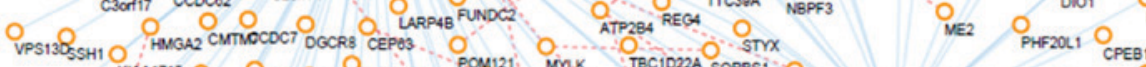

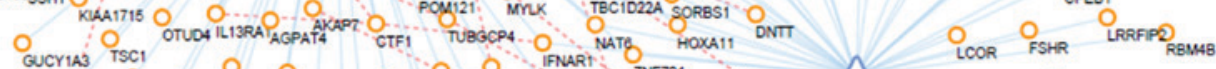

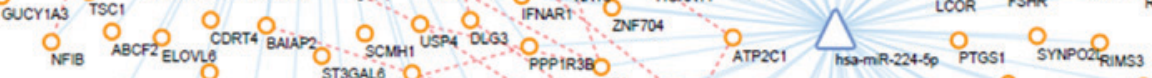

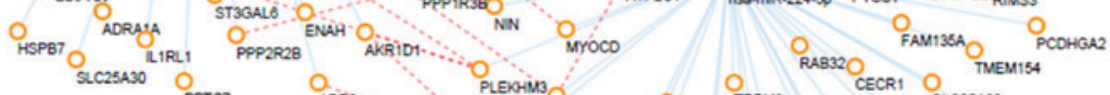

Figure 6. Regulatory network. (A) Regulatory network of upregulated DEGs. (B) regulatory network of downregulated DEGs. Circles represent differentially expressed mRNAs, triangles represent differentially expressed microRNAs, blue lines represent microRNA/mRNA interactions, and orange dotted lines represent protein-protein interactions. DEGs, differentially expressed genes. 
A

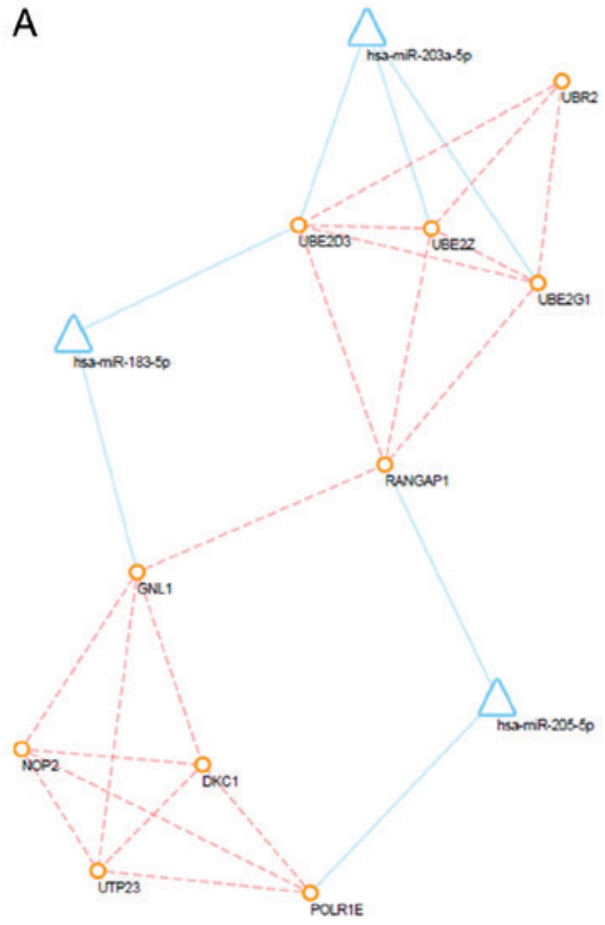

B

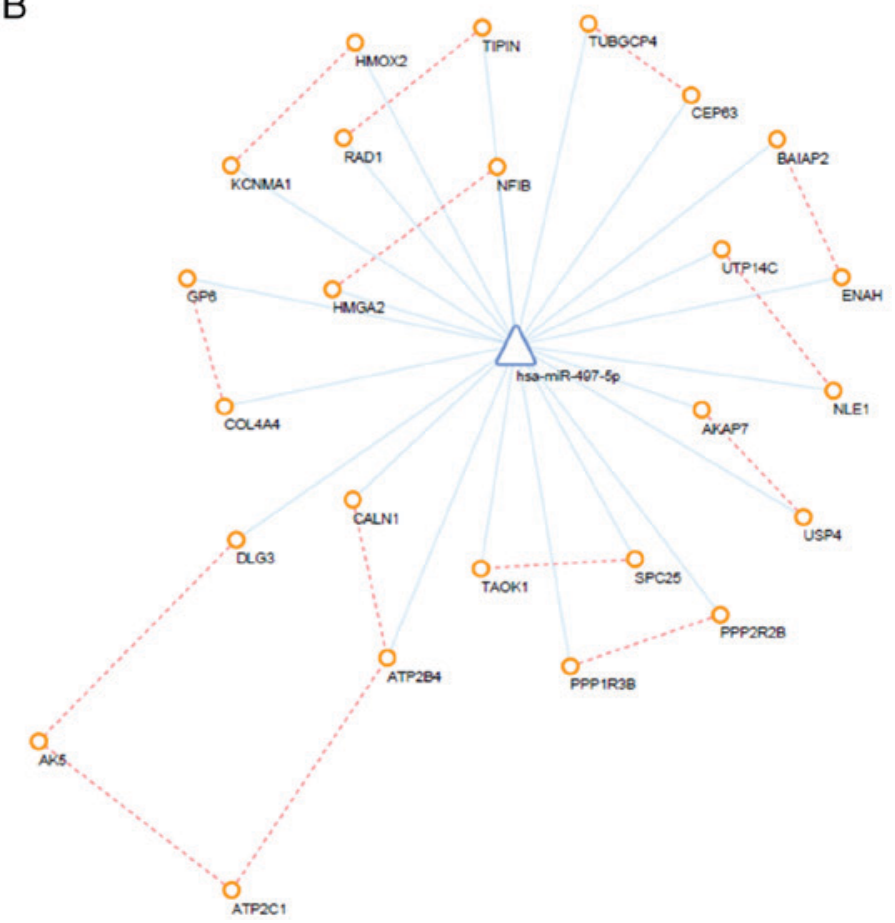

Figure 7. Sub-regulatory network. (A) Sub-regulatory network of upregulated differentially expressed genes. (B) Sub-regulatory network of downregulated DEGs. Circles represent differentially expressed mRNAs, triangles represent differentially expressed microRNAs, blue lines represent microRNA/mRNA interactions, orange dotted lines represent protein-protein interactions.
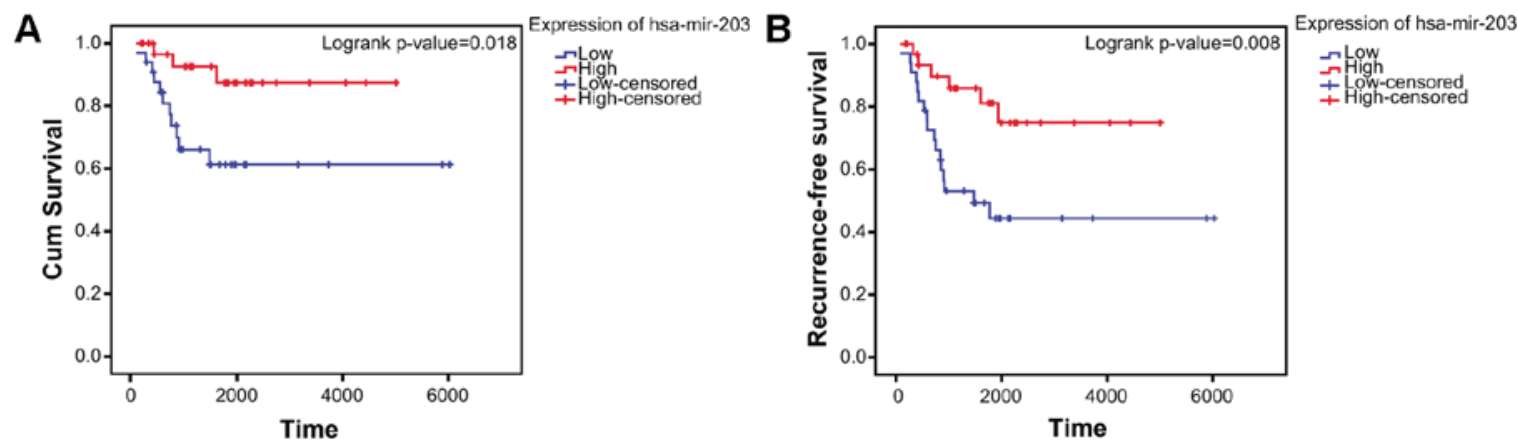

Figure 8. Survival analysis. (A) Kaplan-Meier analysis of hsa-mir-203 expression level and overall survival of patients with osteosarcoma. (B) Kaplan-Meier analysis of hsa-mir-203 expression level and recurrence rate of patients with osteosarcoma. hsa, Homo sapiens; miR, microRNA.

UBE2D3, UBE2Z and RANGAP1, which were enriched in UPP, may serve a negative role in the development and progression of cancer; the novel association between UBE2G1 and UBE2Z and cancer requires further investigation. Liu and Feng (21) reported that miR-203 was downregulated in OS tissues and cell lines, and was associated with poor survival of patients with OS. Most studies suggest that hsa-miR-203 acts as a tumor suppressor by repressing tumor growth and invasion, and downregulating hsa-miR-21; overexpression of hsa-miR-21 is described in several reported carcinogenic processes, such as invasion and metastasis and is closely associated with poor survival (21-23). However, Ikenaga et al (24) reported that hsa-miR-203 was overexpressed in pancreatic adenocarcinoma and was an independent predictor of poor prognosis in patients with pancreatic adenocarcinoma. These findings indicate that hsa-miR-203 has a complex role in cancer initiation and progression as miR-203 interacts with may mRNA targets.
Thus, the precise mechanism of hsa-miR-203 in tumorigenesis and progression requires further research.

Hypoxia, which is associated with tumorigenesis, tumor development, invasion and metastasis, commonly occurs in rapidly growing solid tumors, while some studies have suggested that hypoxia is a common phenomenon in OS $(25,26)$. Accumulating evidence suggests that hypoxia promotes tumorigenesis, tumor progression, invasion and migration (26-28). Heme oxygenase 2 and potassium calcium-activated channel subfamily $\mathrm{M} \alpha 1$ (KCNMA1), the downregulated DEM that was associated with response to hypoxia, may be potential target mRNAs of hsa-miR-497 and was both involved in potassium transport. It has been reported that miR-497, a hypoxia-inducible miRNA, which was suppressed in OS when compared with adjacent tissues, inhibits the expression of hypoxia-inducible factor 1- $\alpha$ and certain other genes $(29,30)$. OS samples from patients 
aged $<20$ exhibited higher levels of miR-497 and reduced expression of downstream DEMs involved in the response to hypoxia, thus inhibiting tumor progression, which may be responsible for the improved survival of patients with OS aged $<20$.

The downregulated DEMs of potentially targeted by miR-497 in OS patients aged $<20$ were mainly involved in potassium transport included proteins of the potassium channel complex and transmembrane transporter complex, such as the Big Potassium (BK) ion channel, also termed KCNMA1, encoded by the DEM KCNMA1. BK channels in biological membranes facilitate the efflux of potassium from intracellular stores. Many ion channels promote cell proliferation, in including the BK channel (31-33). Several reports have demonstrated that BK channels have active roles in tumor growth and metastasis, and the development of drug resistance in glioma, breast cancer and prostate cancer (34-36); whereas Cambien et al (37) contradicted this by reporting that the genetic knockdown of BK $\alpha$ promoted OS progression. Furthermore, BK channel is also reported to promote breast cancer and glioma invasion and migration $(38,39)$; however, there is limited evidence of the role of BK channel in OS invasion and metastasis. The findings of the current study that BK channels have a complex role in cell proliferation with numerous other factors involved. In the present study, BK channel-related genes were significantly downregulated in OS patients aged $<20$, who exhibited better prognosis. Thus, we hypothesized that the active BK channel may partly contribute to poorer survival.

Age is considered to be a prognostic factor in patients with OS; however, the differences in the molecular mechanisms of OS according to age are unclear. In the current study a regulatory network for OS was created by combining PPI network and miRNA-mRNA interactions, and finally we extracted the key subnetwork by topologic analysis. The study provides some insightful information to interpret why younger patients with OS have a far more positive outcome. Furthermore, certain genes may act as potential prognostic factors and effective drug targets for treatment were identified. However, further research is required to determine the exact roles of the identified genes in OS; further investigation into the role other hub miRNAs, including hsa-miR-497, hsa-miR-183 and hsa-miR-205 in the development of OS should be conducted in the future.

\section{Acknowledgements}

Not applicable.

\section{Funding}

This study was supported by the Foundation of Shenzhen Health and Family Planning Commission (grant no. SZFZ2017081).

\section{Availability of data and materials}

The datasets we utilized are available in the GEO online database (accession number no. GSE39058 and GSE39040). https://www.ncbi.nlm.nih.gov/geo/query/acc.cgi?acc=GSE39058; https://www.ncbi.nlm.nih.gov/geo/query/acc.cgi?acc=GSE39040.

\section{Authors' contributions}

JMH and JSW designed the study. JSW analysed and interpreted the microarray profile downloaded from GEO database. MYD, SXD, PX and GZZ made substantial contributions to data analysis. JSW and MYD wrote the manuscript. YSZ and XDL performed Kaplan-Meier survival analysis. JMH was primarily responsible for writing the manuscript. All authors read and approved the final manuscript.

\section{Ethics approval and consent to participate}

Not applicable.

\section{Patient consent for publication}

Not applicable.

\section{Competing interests}

The authors declare that they have no competing interests.

\section{References}

1. Sugalski AJ, Jiwani A, Ketchum NS, Cornell J, Williams R, Heim-Hall J, Hung JY and Langevin AM: Characterization of localized osteosarcoma of the extremity in children, adolescents, and young adults from a single institution in South Texas. J Pediatr Hematol Oncol 36: e353-e358, 2014.

2. Mirabello L, Troisi RJ and Savage SA: Osteosarcoma incidence and survival rates from 1973 to 2004: Data from the surveillance, epidemiology, and end results program. Cancer 115: 1531-1543, 2009.

3. Bleyer A, O'Leary M, Barr R, Ries LAG (eds): Cancer Epidemiology in Older Adolescents and Young Adults 15 to 29 Years of Age, Including SEER Incidence and Survival: 1975-2000. NIH Pub. No. 06-5767. National Cancer Institute, Bethesda, MD, 2006.

4. Hagleitner MM, Hoogerbrugge PM, van der Graaf WT, Flucke U, Schreuder HW and te Loo DM: Age as prognostic factor in patients with osteosarcoma. Bone 49: 1173-1177, 2011.

5. Bielack SS, Kempf-Bielack B, Delling G, Exner GU, Flege S, Helmke K, Kotz R, Salzer-Kuntschik M, Werner M, Winkelmann W, et al: Prognostic factors in high-grade osteosarcoma of the extremities or trunk: An analysis of 1,702 patients treated on neoadjuvant cooperative osteosarcoma study group protocols. J Clin Oncol 20: 776-790, 2002.

6. Bacci G, Longhi A, Versari M, Mercuri M, Briccoli A and Picci P: Prognostic factors for osteosarcoma of the extremity treated with neoadjuvant chemotherapy: 15 -year experience in 789 patients treated at a single institution. Cancer 106: 1154-1161, 2006.

7. Whelan JS, Jinks RC, McTiernan A, Sydes MR, Hook JM, Trani L, Uscinska B, Bramwell V, Lewis IJ, Nooij MA, et al: Survival from high-grade localised extremity osteosarcoma: Combined results and prognostic factors from three European Osteosarcoma Intergroup randomised controlled trials. Ann Oncol 23: 1607-1616, 2012.

8. Kelly AD, Haibe-Kains B, Janeway KA, Hill KE, Howe E, Goldsmith J, Kurek K, Perez-Atayde AR, Francoeur N, Fan JB, et al: MicroRNA paraffin-based studies in osteosarcoma reveal reproducible independent prognostic profiles at $14 \mathrm{q} 32$. Genome Med 5: 2, 2013.

9. Diboun I, Wernisch L, Orengo CA and Koltzenburg M: Microarray analysis after RNA amplification can detect pronounced differences in gene expression using limma. BMC Genomics 7: 252, 2006.

10. Ritchie ME, Phipson B, Wu D, Hu Y, Law CW, Shi W and Smyth GK: Limma powers differential expression analyses for RNA-sequencing and microarray studies. Nucleic Acids Res 43: e47, 2015 .

11. Wickham H, ggplot2: Elegant graphics for data analysis. Springer-Verlag, New York, NY, 2016. 
12. Scardoni G, Petterlini M and Laudanna C: Analyzing biological network parameters with CentiScaPe. Bioinformatics 25: 2857-2859, 2009.

13. Bader GD and Hogue CW: An automated method for finding molecular complexes in large protein interaction networks. BMC Bioinformatics 4: 2, 2003.

14. Eldeeb MA and Fahlman RP: The anti-apoptotic form of tyrosine kinase Lyn that is generated by proteolysis is degraded by the N-end rule pathway. Oncotarget 5: 2714-2722, 2014

15. Zhang G, Lin RK, Kwon YT and Li YP: Signaling mechanism of tumor cell-induced up-regulation of E3 ubiquitin ligase UBR2. FASEB J 27: 2893-2901, 2013.

16. Lin TY, Lee CC, Chen KC, Lin CJ and Shih CM: Inhibition of RNA transportation induces glioma cell apoptosis via downregulation of RanGAP1 expression. Chem Biol Interact 232: 49-57, 2015.

17. Mao J, Liang Z, Zhang B, Yang H, Li X, Fu H, Zhang X, Yan Y, $\mathrm{Xu}$ W and Qian H: UBR2 enriched in p53 deficient mouse bone marrow mesenchymal stem cell-exosome promoted gastric cancer progression via Wnt/beta-catenin pathway. Stem Cells 35: 2267-2279, 2017

18. Guan GG, Wang WB, Lei BX, Wang QL, Wu L, Fu ZM, Zhou FX and Zhou YF: UBE2D3 is a positive prognostic factor and is negatively correlated with hTERT expression in esophageal cancer. Oncol Lett 9: 1567-1574, 2015.

19. Wang W, Yang L, Hu L, Li F, Ren L, Yu H, Liu Y, Xia L, Lei H, Liao Z, et al: Inhibition of UBE2D3 expression attenuates radiosensitivity of MCF-7 human breast cancer cells by increasing hTERT expression and activity. PLoS One 8: e64660, 2013.

20. Gao X, Wang W, Yang H, Wu L, He Z, Zhou S, Zhao H, Fu Z, Zhou $\mathrm{F}$ and Zhou Y: UBE2D3 gene overexpression increases radiosensitivity of EC109 esophageal cancer cells in vitro and in vivo 7: 32543-32553, 2016.

21. Liu S and Feng P: MiR-203 determines poor outcome and suppresses tumor growth by targeting TBK1 in osteosarcoma. Cell Physiol Biochem 37: 1956-1966, 2015.

22. Zhang F, Yang Z, Cao M, Xu Y, Li J, Chen X, Gao Z, Xin J, Zhou S, Zhou Z, et al: MiR-203 suppresses tumor growth and invasion and down-regulates MiR-21 expression through repressing Ran in esophageal cancer. Cancer Lett 342: 121-129, 2014.

23. Krichevsky AM and Gabriely G: miR-21: A small multi-faceted RNA. J Cell Mol Med 13: 39-53, 2009.

24. Ikenaga N, Ohuchida K, Mizumoto K, Yu J, Kayashima T, Sakai H, Fujita H, Nakata K and Tanaka M: MicroRNA-203 expression as a new prognostic marker of pancreatic adenocarcinoma. Ann Surg Oncol 17: 3120-3128, 2010.

25. Kunz M and Ibrahim SM: Molecular responses to hypoxia in tumor cells. Mol Cancer 2: 23, 2003.

26. Guo M, Cai C, Zhao G, Qiu X, Zhao H, Ma Q, Tian L, Li X, Hu Y and Liao B: Hypoxia promotes migration and induces CXCR4 expression via HIF-1alpha activation in human osteosarcoma. PLoS One 9: e90518, 2014
27. Guan G, Zhang Y, Lu Y, Liu L, Shi D, Wen Y, Yang L, Ma Q, Liu T, Zhu X, et al: The HIF-1alpha/CXCR4 pathway supports hypoxia-induced metastasis of human osteosarcoma cells. Cancer Lett 357: 254-264, 2015.

28. Cao J, Wang Y, Dong R, Lin G, Zhang N, Wang J, Lin N, Gu Y, Ding L, Ying M, et al: Hypoxia-Induced WSB1 promotes the metastatic potential of osteosarcoma cells. Cancer Res 75: 4839-4851, 2015

29. Shan K, Pang R, Zhao C, Liu X, Gao W, Zhang J, Zhao D, Wang Y and Qiu W: IL-17-triggered downregulation of miR-497 results in high HIF-1alpha expression and consequent IL-1beta and IL-6 production by astrocytes in EAE mice. Cell Mol Immunol, 2017.

30. Wu Z, Cai X, Huang C, Xu J and Liu A: miR-497 suppresses angiogenesis in breast carcinoma by targeting HIF-1alpha. Oncol Rep 35: 1696-16702, 2016.

31. Hoffmann EK and Lambert IH: Ion channels and transporters in the development of drug resistance in cancer cells. Philos Trans R Soc Lond B Biol Sci 369: 20130109, 2014.

32. Weaver AK, Liu X and Sontheimer H: Role for calcium-activated potassium channels (BK) in growth control of human malignant glioma cells. J Neurosci Res 78: 224-234, 2004.

33. Urrego D, Tomczak AP, Zahed F, Stühmer W and Pardo LA: Potassium channels in cell cycle and cell proliferation. Philos Trans R Soc Lond B Biol Sci 369: 20130094, 2014

34. Bloch M, Ousingsawat J, Simon R, Schraml P, Gasser TC, Mihatsch MJ, Kunzelmann K and Bubendorf L: KCNMA1 gene amplification promotes tumor cell proliferation in human prostate cancer. Oncogene 26: 2525-2534, 2007.

35. Oeggerli M, Tian Y, Ruiz C, Wijker B, Sauter G, Obermann E, Güth U, Zlobec I, Sausbier M, Kunzelmann K and Bubendorf L: Role of KCNMA1 in breast cancer. PLoS One 7: e41664, 2012.

36. Bury M, Girault A, Mégalizzi V, Spiegl-Kreinecker S, Mathieu V, Berger W, Evidente A, Kornienko A, Gailly P, Vandier C and Kiss R: Ophiobolin A induces paraptosis-like cell death in human glioblastoma cells by decreasing BKCa channel activity. Cell Death Dis 4: e561, 2013.

37. Cambien B, Rezzonico R, Vitale S, Rouzaire-Dubois B, Dubois JM, Barthel R, Soilihi BK, Mograbi B, Schmid-Alliana A and Schmid-Antomarchi H: Silencing of hSlo potassium channels in human osteosarcoma cells promotes tumorigenesis. Int J Cancer 123: 365-371, 2008.

38. Khaitan D, Sankpal UT, Weksler B, Meister EA, Romero IA, Couraud PO and Ningaraj NS: Role of KCNMA1 gene in breast cancer invasion and metastasis to brain. BMC Cancer 9: 258, 2009.

39. Sontheimer $\mathrm{H}$ : An unexpected role for ion channels in brain tumor metastasis. Exp Biol Med (Maywood) 233: 779-791, 2008.

This work is licensed under a Creative Commons Attribution-NonCommercial-NoDerivatives 4.0 International (CC BY-NC-ND 4.0) License. 